\title{
On the Phononic Bandgap of Carbon Nanotubes
}

\author{
Kohei Yamamoto, ${ }^{1}$ Hiroyuki Ishii, ${ }^{1}$ Nobuhiko Kobayashi, ${ }^{1}$ and Kenji Hirose ${ }^{2}$ \\ ${ }^{1}$ Institute of Applied Physics and Tsukuba Research Center for Interdisciplinary Materials Science, \\ University of Tsukuba, 1-1-1 Tennoudai, Tsukuba, Ibaraki 305-8573, Japan \\ ${ }^{2}$ Green Innovation Research Laboratory, NEC Corporation, 34 Miyukigaoka, Tsukuba, Ibaraki 305-8501, Japan \\ Correspondence should be addressed to Nobuhiko Kobayashi; nkoba@bk.tsukuba.ac.jp
}

Received 14 April 2013; Accepted 4 June 2013

Academic Editor: Nadya Mason

Copyright (C) 2013 Kohei Yamamoto et al. This is an open access article distributed under the Creative Commons Attribution License, which permits unrestricted use, distribution, and reproduction in any medium, provided the original work is properly cited.

On the phononic bandgap of carbon nanotubes (CNTs), we show in what chirality CNTs have phononic bandgaps and its dependence on the diameters of CNTs. We find that, though the rule where CNTs have phononic bandgaps is the same as in the electronic structure case, the diameter dependence is different. The phononic bandgaps of the zigzag-CNTs reveal "three" kinds of diameter dependence due to the anisotropy of graphene phonon band around the K point in $k$-space. We also show the crossover from one- to two-dimensional characteristics in phononic bandgaps.

Recently phononic bandgaps attract much attention. Following an analogy of the photonic crystals, in the last decade, artificial crystals with specific structures have been studied systematically to construct the phononic bandgaps since periodic structures might exhibit bandgaps in the band structures for the wave propagation [1]. Since phonon waves cannot propagate in the phononic bandgaps, the materials with effective phononic bandgaps become candidates for the applications of phonon frequency filter (Figure 1(a)), phonon beam splitter, phonon waveguide [2], and sound or vibration protection devices and thermopower waves [3]. Also phononic bandgaps play important roles in view of the reduction of thermal phonon transport, which leads to the effective heat managements [4]. Thus the control and management of phononic bandgaps are very important for various applications and have been widely studied [5].

In this respect, Sigmund and Jensen [6] studied systematic design of phononic bandgaps with topology optimization and Gazonas et al. [7] studied the optimization of phononic bandgap structures using the genetic algorithm. They dealt with sonic or ultrasonic crystals which have midgap frequencies $\omega_{g}=10^{2}-10^{6} \mathrm{~Hz}$. Gorishnyy et al. [8] fabricated hypersonic phononic crystals with lithography technique, and Cheng et al. [9] constructed and tuned hypersonic bandgaps in colloidal crystals which have $\omega_{g}=10^{9}-10^{12} \mathrm{~Hz}$.
These phonon bandgap engineerings deal with the artificially made phononic crystals. To obtain phononic bandgaps on the higher frequency requires higher skills and techniques for the fabrication, and thus materials which can be easily synthesized and to exhibit selectable hypersonic phononic bandgaps are desired. In this paper, we show that carbon nanotubes (CNTs) [10], which are easily synthesizable, have highest phononic bandgaps with $\omega_{g} \sim 10^{13} \mathrm{~Hz}$. Furthermore the magnitude of phononic bandgaps of CNTs changes from $0 \mathrm{THz}$ to $2 \mathrm{THz}$ according to the diameters.

So far, the electronic structures of CNTs have been studied and shown to exhibit fascinating properties such as extremely high electric conductivity. The band structures of CNTs show appearance and disappearance of bandgaps at the Fermi level depending on the diameter and chirality. Correspondingly, transport properties of CNTs change from metallic to semiconducting ones according to the magnitude of bandgaps [11, 12]. The emergence of bandgaps in the electronic structures can be explained in view of the electronic states of twodimensional (2D) graphene. While bandgaps in the electronic states of CNTs are well known, those of the phononic band structures are not clearly understood. Here, we focus on the phononic bandgap problem of CNTs.

The phononic band structures of CNTs and graphene are calculated with use of the Brenner interatomic potential [13]. 


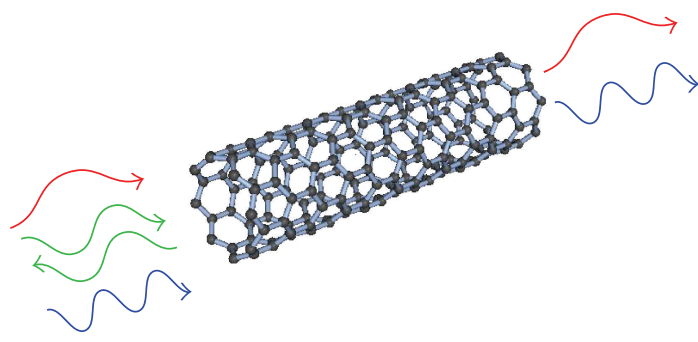

(a)

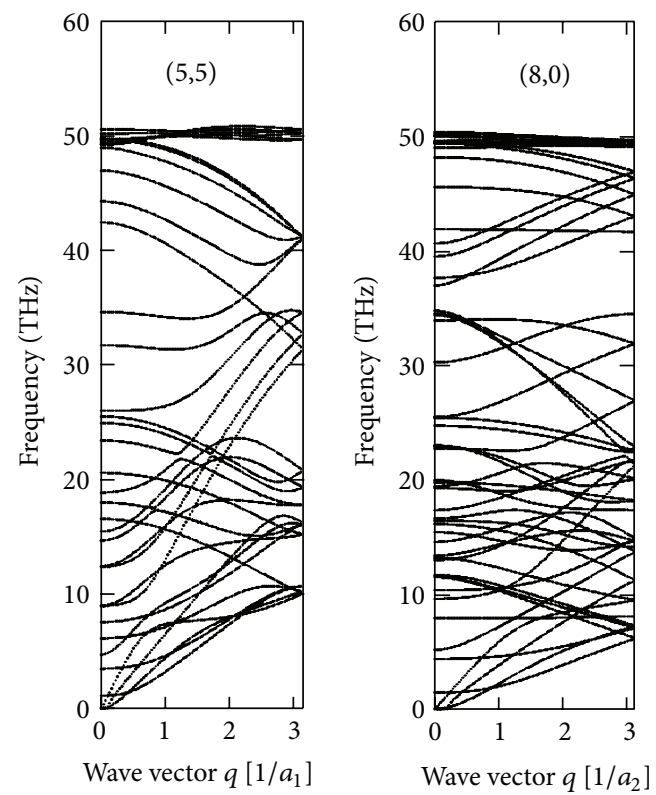

(b)

Figure 1: (a) Schematic view of $(8,0)$ CNT for an application of elastic wave filter in the particular frequency around $36 \mathrm{THz}$. (b) Phonon band structures of CNTs with chirality $(5,5)$ (left) and $(8,0)$ (right). We see that $(5,5) \mathrm{CNT}$ exhibits no phononic bandgap up to $50 \mathrm{THz}$, while $(8,0)$ CNT has a phononic bandgap around $36 \mathrm{THz}$, corresponding to $150 \mathrm{meV}$. Here $a_{1}$ and $a_{2}$ are unit cell lengths for $(5,5) \mathrm{CNT}$ and $(8,0) \mathrm{CNT}$, respectively.

The coordinates of carbon atoms of CNTs are constructed by the rolling of graphene sheet, for which the interatomic distances are optimized by the total energy minimization in the Brenner potential. The force constants are constructed in the optimization process by finite difference of the interatomic forces, which are calculated from differentiation of the total energy. The phonon dispersion relations of the system are obtained by the diagonalization of the dynamical matrix, which is constructed from the force constants [14].

Figure $1(\mathrm{~b})$ show the phonon bands of $(5,5)$ and $(8,0)$ CNTs. We see that there are no phononic bandgaps in the phonon band structure of $(5,5) \mathrm{CNT}$, while $(8,0) \mathrm{CNT}$ has a phononic bandgap around the frequency of $36 \mathrm{THz}$. This shows that phononic bandgap appears in this quasione-dimensional nanometer-scale carbon material. Then we would like to ask what is the condition that CNTs have phononic bandgaps in the phonon bands and the relation

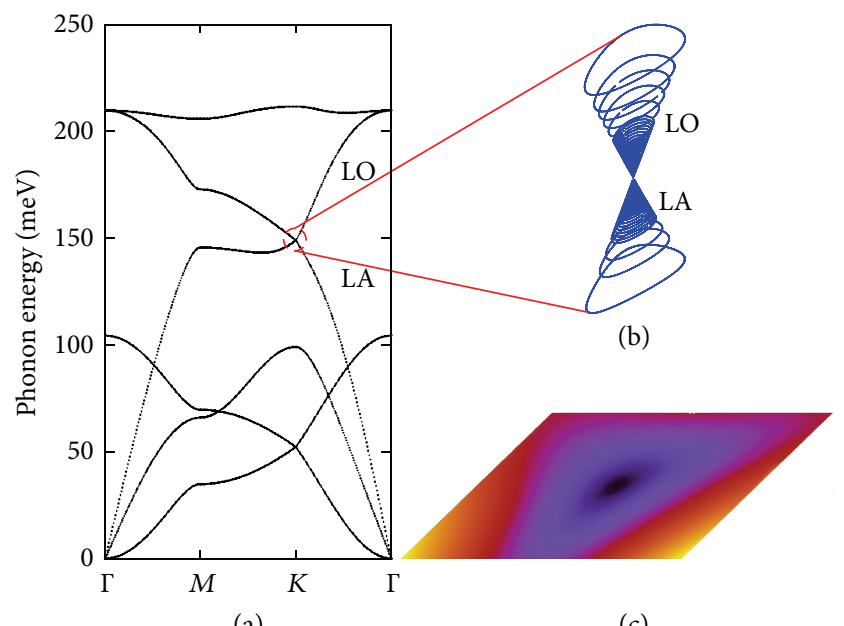

(a)

(c)

FIGURE 2: (a) Phonon band of graphene: the LA-mode tangents to the LO-mode at the $\mathrm{K}$ point. There are only LA and LO modes around $150 \mathrm{meV}$ corresponding to the center of phononic bandgap of CNTs. (b) Blue lines are contours near the K point with equal phonon energy in the phonon band of graphene. Dirac's cone is isotropic close to the $\mathrm{K}$ point and becomes distorted away from the K point. (c) Surface plot of energy difference between LA and LO modes. Center black region indicates no energy difference at the $\mathrm{K}$ point. As the color becomes warmer, the energy difference becomes larger.

of magnitudes of phononic bandgaps with the diameters of CNTs.

Figure 2(a) shows the phonon band of graphene. We see that there are only two modes around $150 \mathrm{meV}$, and these two modes are crossed at the $\mathrm{K}$ point in $k$-space. These two correspond to the longitudinal acoustic (LA) mode and the longitudinal optical (LO) mode, and no other phonon bands are present. We note that this situation is very close to the electronic band structure of graphene around the Fermi level, where two kinds of linear-dispersion bands, bonding and anti-bonding $\pi$ bands, are crossed at the $\mathrm{K}$ point. This shows that the $\mathrm{K}$ point is a special point also in the phonon band as in the electronic band. This evidence leads us to the conclusion that, as in the electronic bands of CNTs, whether the phonon bands have finite phononic bandgaps or not is determined by the conditions that the phonon bands of graphene crosses the $\mathrm{K}$ point or not by the rolling up to construct the CNTs. In the electronic band structures, the zone-folding method [15] reveals that bandgaps of CNTs are made from the band of graphene at wave vectors selected by the periodic boundary condition along the circumference direction on CNTs.

The same method is applied to the case of phononic bandgaps. CNTs with the index $n-m$ equal to the multiple of 3 have no phononic bandgaps. Thus the armchair CNTs with $(n, n)$ chirality have no phononic bandgap, because the wave vector of phonon bands of the armchair CNTs always cross the $\mathrm{K}$ point of phonon bands of graphene. This is observed in Figure 1 (left), which shows the phonon band of $(5,5)$ armchair CNT. On the other hand, the phonon bands of 


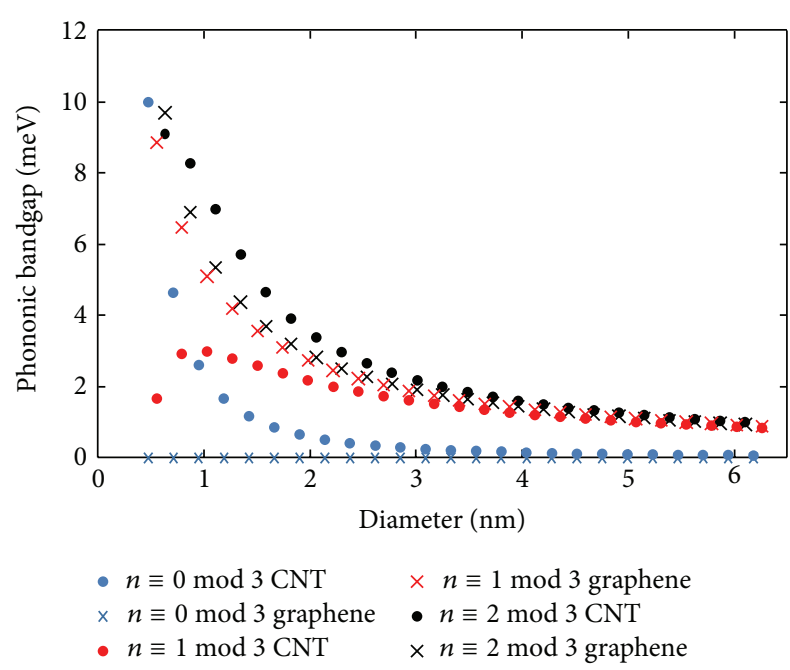

FIGURE 3: Diameter dependence of phononic bandgaps of zigzagCNTs with chirality $(n, 0)$ from $n=6$ to 77 . Dots show phononic bandgaps from the phonon bands, and crosspoints show those by the zone-folding method. Blue colors are phononic bandgaps where $n$ is the multiple of 3 , red colors are those where $n$ is the sum of multiples of 3 and 1, and black colors are those where $n$ is the sum of multiple of 3 and 2 .

the zigzag CNTs with $(n, 0)$ chirality in Figure 1 (right) have finite phononic bandgap.

Figure 3 shows the magnitudes of phononic bandgaps of the zigzag CNTs for various diameters and chirality $(n, 0)$ with $n$ from 6 to 77 . Dots show the phononic bandgaps obtained from the direct calculations of phonon bands, while the cross points show those by the zone-folding method, extraction from the graphene phonon band according to the wave vector selection rule on the periodic boundary conditions. We see that there are "three" kinds of diameter dependences of phononic bandgaps. Note that only two kinds of diameter dependences are present for the bandgaps of electronic states.

This difference is caused by the anisotropy of phononic bands of graphene around the K point. When $n$ is the sum of multiples of 3 and 1 , the allowed wave vector near the K point in the zone-folding method approaches the $\mathrm{K}$ point from the $\mathrm{M}$ point. On the other hand, when $n$ is the sum of multiples of 3 and 2, the allowed wave vector approaches the $\mathrm{K}$ point from the $\Gamma$ point. Along these two different directions to the $\mathrm{K}$ point, we can clearly see the anisotropy of phonon band in Figure 2(a). The anisotropic phonon band structure, different from the linear dispersion of electronic band structure, leads to "three" kinds of diameter dependences of phononic bandgaps. As the diameters of CNTs become large, two kinds of allowed wave vectors from the $M$ and $\Gamma$ points to the $K$ point become so small enough to ignore the anisotropy of phonon bands of graphene around the $\mathrm{K}$ point and then the magnitudes of phononic bandgaps become the same. In the phonon bands of graphene close to the K point in Figures 2(b) and 2(c), we see that the Dirac's cone near the K point is isotropic, while the cone becomes distorted far from the $\mathrm{K}$ point. This leads to the diameter dependences of phononic bandgaps to change from "three" kinds in the small $n$ to two kinds in the large $n$.

In Figure 3, we see that dots deviate from cross points for small $n$ with small diameters. For instance, when $n$ is the multiple of 3 , there are no bandgaps in cross points, while small phononic bandgaps appear in dots, which are directly calculated from the phonon bands. The difference of phononic bandgaps between two methods is also seen for the electronic bandgap calculation [11]. Tight-binding calculations of electronic band structure for $(n, 0)$ zigzag CNTs when $n$ is the multiple of 3 exhibit narrow bandgaps due to the nonorthogonality conditions of the atomic orbitals, and displacement of the atomic points where bonding and antibonding $\pi$ bands are degenerate. Namely, the difference between two methods is caused by the curvature of CNTs.

As the diameter of CNT becomes large, the effects become less important and the phononic bandgaps of CNTs with large diameter is explained in terms of the phonon band structure of graphene. In this respect, the deviation of two methods reveals the crossover from one-dimensional to two-dimensional characteristics. Let us see two special diameters for this crossover. First, we consider the optimized interatomic distance. For CNTs with small diameters, the distances between carbon atoms become longer than that of graphene. We find that, for $n>16$ with the diameter of $1.3 \mathrm{~nm}$, the distance of carbon atoms in CNTs agree with that of graphene, showing the crossover from tube like to sheet like. Second, a more minor point, CNTs have specific phonon dispersion relations close to the $\Gamma$ point where acoustic phonon branches have special properties with zero frequencies. For one-dimensional material, it is known that phonon bands have four acoustic phonon branches, while only three acoustic phonon branches exist for two- and threedimensional materials. We find that CNTs with $n>35$ have only three acoustic phonons. This leads to the small deviation of phononic bandgaps of CNTs with diameters larger than about $3 \mathrm{~nm}$ in the zone-folding method as is seen in Figure 3. These show that, for CNTs which have diameter smaller than $1.3 \mathrm{~nm}$, phonon bandgaps must be calculated directly from the phonon bands of CNTs.

To summarize, we study the phononic bandgap of CNTs. We find that the condition of chirality of CNTs which have finite phononic bandgaps is almost the same as that of the electronic bandgap case. This is due to the fact that phonon band of graphene has only two bands close to the $\mathrm{K}$ point at specific energy around $150 \mathrm{meV}$, while electronic band structure of graphene has only two bands around the $\mathrm{K}$ point at the Fermi level. Diameter dependence of phononic bandgaps is, however, quite different. We find that phononic bandgap has "three" kinds of diameter dependence, different from the electronic bandgap which has only two kinds. This difference is caused by the anisotropy of the Dirac's cone around the K point of graphene. While the Dirac's cone is isotropic close to the $\mathrm{K}$ point for phonon bands of graphene, it becomes distorted away from the $\mathrm{K}$ point. This changes the dependences of phononic bandgaps from three kinds for small diameter to two kinds for large diameter. Due to the curvature of CNTs at small diameter, phononic bandgaps differ with two calculating methods: direct calculation for 
CNTs and zone-folding method for graphene, for which we need the direct one. As the diameter becomes large, curvature effects become less important and the difference becomes small. We find that we need the direct phonon dispersion calculation for diameter smaller than $1.3 \mathrm{~nm}$ and that the zone-folding method becomes reliable for diameter larger than $3 \mathrm{~nm}$. This is regarded as the dimensional crossover from one- to two-dimensional characteristics in phononic bandgaps.

\section{Acknowledgment}

This work is supported in part by a Grant-in-Aid for Scientific Research from the Ministry of Education, Culture, Sports, Science and Technology of Japan.

\section{References}

[1] A. V. Akimov, Y. Tanaka, A. B. Pevtsov et al., "Hypersonic modulation of light in three-dimensional photonic and phononic band-gap materials," Physical Review Letters, vol. 101, no. 3, Article ID 033902, 2008.

[2] C. W. Chang, D. Okawa, H. Garcia, A. Manjumdar, and A. Zettl, "Nanotube phonon waveguide," Physical Review Letters, vol. 99, no. 4, Article ID 045901, 4 pages, 2007.

[3] W. Choi, S. Hong, J. T. Abrahamson et al., "Chemically driven carbon-nanotube-guided thermopower waves," Nature Materials, vol. 9, no. 5, pp. 423-429, 2010.

[4] M. Maldovan, "Narrow low-frequency spectrum and heat management by thermocrystals," Physical Review Letters, vol. 110, no. 2, Article ID 025902, 5 pages, 2013.

[5] T. P. M. Alegre, A. Safavi-Naeini, M. Winger, and O. Painter, "Quasi-two-dimensional optomechanical crystals with a complete phononic bandgap," Optics Express, vol. 19, no. 6, pp. 56585669, 2011.

[6] O. Sigmund and J. S. Jensen, "Systematic design of phononic band-gap materials and structures by topology optimization," Philosophical Transactions of the Royal Society A, vol. 361, no. 1806, pp. 1001-1019, 2003.

[7] G. A. Gazonas, D. S. Weile, R. Wildman, and A. Mohan, "Genetic algorithm optimization of phononic bandgap structures," International Journal of Solids and Structures, vol. 43, no. 18-19, pp. 5851-5866, 2006.

[8] T. Gorishnyy, C. K. Ullal, M. Maldovan, G. Fytas, and E. L. Thomas, "Hypersonic phononic crystals," Physical Review Letters, vol. 94, no. 11, Article ID 115501, 4 pages, 2005.

[9] W. Cheng, J. Wang, U. Jonas, G. Fytas, and N. Stefanou, "Observation and tuning of hypersonic bandgaps in colloidal crystals," Nature Materials, vol. 5, no. 10, pp. 830-836, 2006.

[10] S. Iijima, "Helical microtubules of graphitic carbon," Nature, vol. 354, no. 6348, pp. 56-58, 1991.

[11] N. Hamada, S.-I. Sawada, and A. Oshiyama, "New onedimensional conductors: graphitic microtubules," Physical Review Letters, vol. 68, no. 10, pp. 1579-1581, 1992.

[12] R. Saito, M. Fujita, G. Dresselhaus, and M. S. Dresselhaus, "Electronic structure of chiral graphene tubules," Applied Physics Letters, vol. 60, no. 18, pp. 2204-2206, 1992.

[13] D. W. Brenner, "Empirical potential for hydrocarbons for use in simulating the chemical vapor deposition of diamond films," Physical Review B, vol. 42, no. 15, pp. 9458-9471, 1990.
[14] K. Yamamoto, H. Ishii, N. Kobayashi, and K. Hirose, "Effects of vacancy defects on thermal conduction of silicon nanowire: nonequilibrium green's function approach," Applied Physics Express, vol. 4, Article ID 085001, 3 pages, 2011.

[15] R. Saito, G. Dresselhaus, and M. S. Dresselhaus, Physical Properties of Carbon Nanotubes, chapter 9, Imperial College Press, London, UK, 1998. 

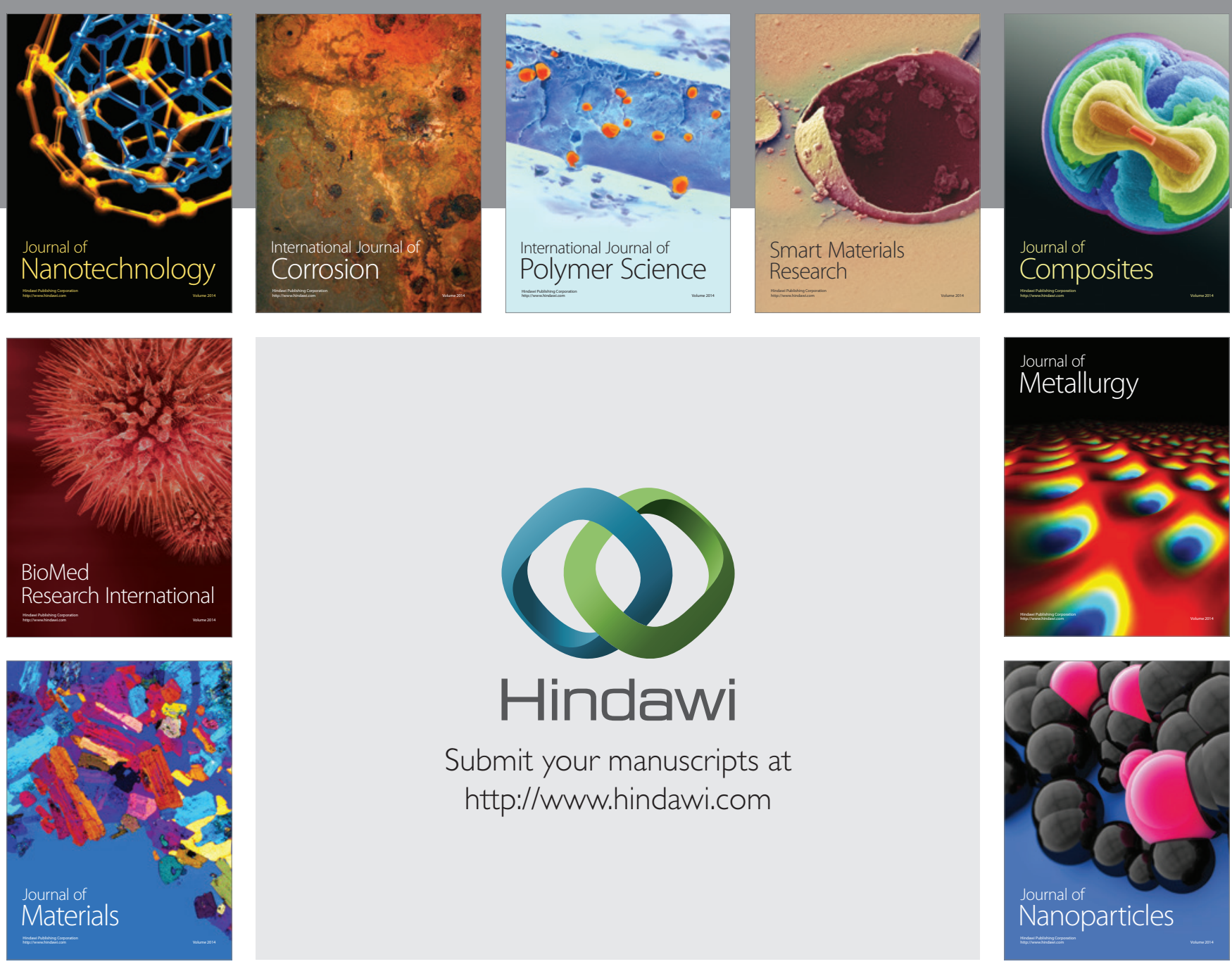

Submit your manuscripts at http://www.hindawi.com
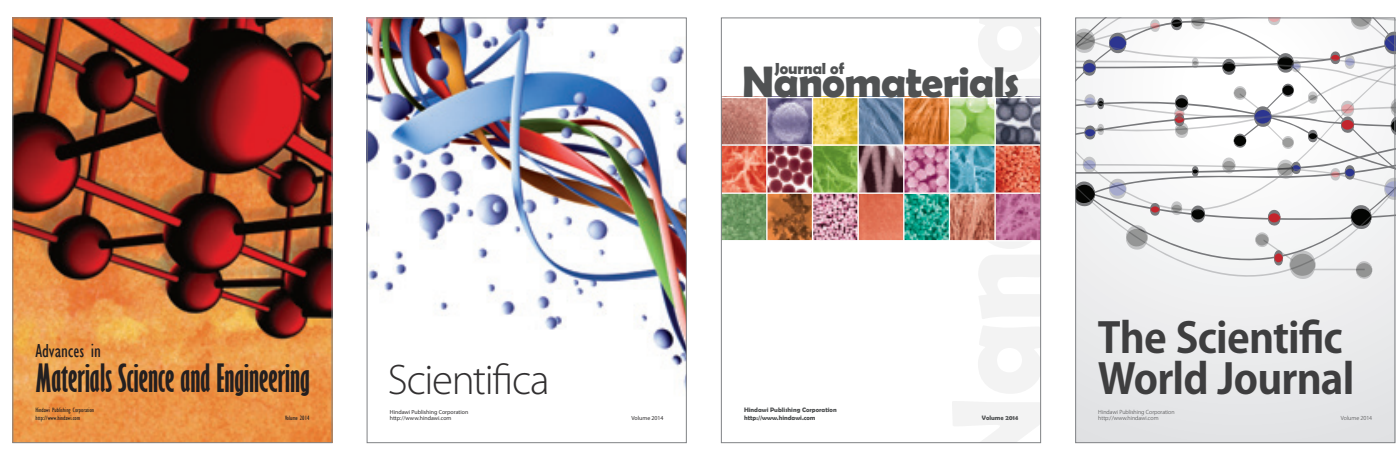

\section{The Scientific World Journal}
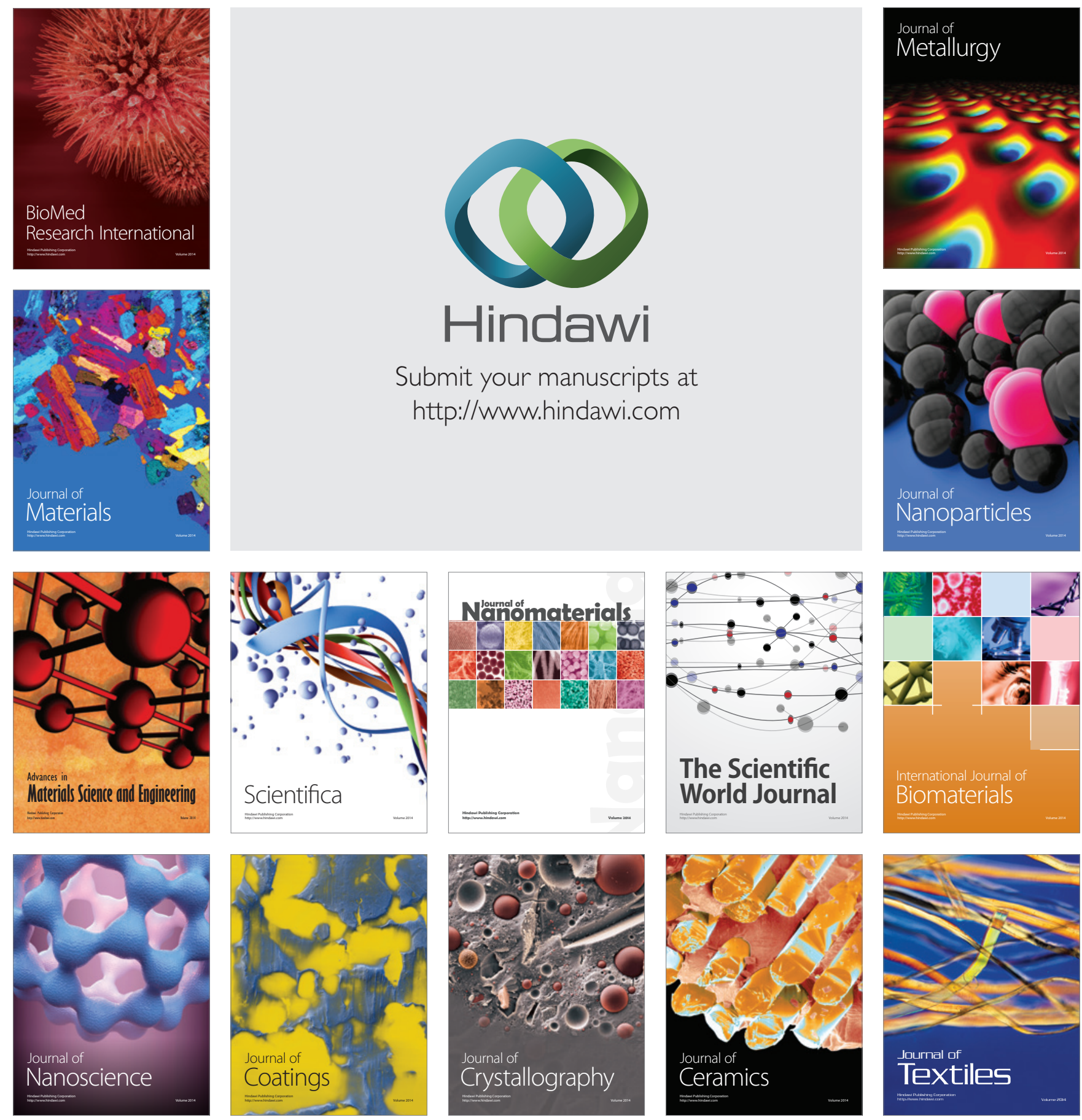